\title{
La visualisation des écoulements en mécanique des fluides
}

\author{
Allocution de M. P. CHAPOUTHTER, \\ PRÉSIDENT-DIRECTEUR GÉNËRAL DE YA S.A.T.E.G.E., \\ PRÉSIDENT DE IA SÉANCE DU 21 MARS 1963 \\ DU COMITÉ TECHNIQUE DE LA SOCIÉTÉ HYDDOTECHNIOUE DE TRANCE
}

Mes chers collègues,

Le développement impressionnant de l'outil mathématique au cours de ces dernières années ne doit pas nous faire oublier que la plus savante déduction n'a jamais remplacé le contact direct avec les choses. Les mathématiciens euxmêmes en conviennent: ils ne peuvent éviter, au départ, des axiomes et des postulats d'origine expérimentale; et quand ils veulent traduire cette perception directe et immédiate des phénomènes, ils emploient volontiers le langage même des empiriques. On les entend dire : «on voit facilement que », « la certitude en est palpable $»$. D'une manière plus savante, ils recourent volontiers au latin. Ils disent : intuere, voir, regarder. Nous avons, des hypothèses de base, de l'espace, du temps, une notion «intuitive».

Vous savez la part prépondérante qu'a chez les ingénieurs l'intuition, la vision directe des choses. Par ce qu'elle a d'exaltant, de stimulant pour l'imagination et pour l'intelligence, elle a toujours jouć un grand rôle pour la Recherche et particulièrement en Hydraulique. Un grand historien des sciences a même pu écrire que «tous les grands hommes qui, depuis le Xvir siècle jusqu'au milieu du xrx" siècle, ont créé l'algèbre, le calcul intégral et la mécanique céleste, ont le plus souvent justifié les plus importantes découvertes à l'aide de raisonnements défectueux ou même de paralogismes flagrants. $\gg(1)$.

Aussi le contrôle du mathématicien est-il constamment nécessaire pour assurer la démar-

(1) Pierre Duhem. La science allemande, 1915. che de l'intuition et légitimer les transpositions qu'elle opère.

Une des tâches essentielles accomplies par les mathématiciens dans la seconde moitié du $\mathrm{XIX}^{\mathrm{c}}$ siècle a été de reprendre les ouvres de leurs devanciers pour compléter et rectifier leurs raisonnements, pour leur montrer - le mot est d'Augustin Cauchy - « comment ils auraient dû trouver ce qu'ils avaient si bien inventé ». Je pense d'ailleurs qu'Augustin Cauchy se trompe et que, par des moyens rigoureux, ils n'auraient jamais inventé.

Un premier service que le mathématicien rend au physicien, c'est de lui permettre de changer les phénomènes, pourvu que les équations qui les régissent restent les mếmes. On sait le développement pris ces dernières années par la méthode des analogies.

Premier exemple: l'écoulement d'un fluide visqueux.

Il est régi, comme vous le savez, par l'équation de Navier. Si, dans cette équation, on peut négliger le terme d'inertie, c'est-à-dire si les vitesses sont faibles, l'équation de Navier devient l'équation de Darcy. C'est l'analogie de HeleShaw, du nom du savant qui l'a découverte en 1898, et dont vont nous parler MM. LE Fur et Aguirre-Puente. Cette analogie visualise en quelque sorte le milieu continu fictif, caractérisé par sa perméabilité géométrique, que l'hydraulique souterraine a pris I'habitude de substituer à la discontinuité réelle du milieu poreux.

Mais on peut aussi changer le fluide : on peut remplacer l'eau par l'électricité. Ce sont alors toutes les analogies électriques, dont vous con- 
naissez l'important développement. On peut encore remplacer un liquide par un gaz ou inversement. J'ai noté avec un certain plaisir, en lisant la seconde communication, celle de M. WErcé, ingénieur de recherches à l'Office national d'Etudes et de Recherches aéronautiques, qu'alors que, pour les hydrauliciens, le dégrossissage de certains phénomènes à l'air a été considéré sinon comme un progrès, du moins comme une économie, pour les aérodynamiciens, c'est souvent l'inverse : un certain nombre de problèmes posés par les gaz sont utilement dégrossis à l'eau. C'est un agréable échange de bons procédés.

On sait en effet que l'écoulement en surface libre d'un fluide incompressible est régi par les mêmes équations que l'écoulement en charge d'un gaz compressible; d'où le passage de l'un à l'autre.

Mais le physicien intervient à son tour pour développer nos moyens d'investigation.

L'œil humain est un outil imparfait. Il est notamment atteint d'une double infirmité, spatiale et temporelle.

Nous ne distinguons pas en decà du $1 / 2000^{\circ}$ de radiant, ni en deçà du $1 / 20^{\circ}$ de seconde. Bienheureuses infirmités qui rendent possibles les joies du cinéma et de la télévision! Mais cette insuffisance de nos moyens physiques ne nous permet pas une analyse assez fine de certains phénomènes.

Deux ingénieurs du Centre Nucléaire de Grenoble, MM. SÉméria el Béhar, vont nous aider, pour les phénomènes d'ébullition et d'écoulements diphasés, à nous affranchir de nos infirmités par trois moyens; et nous pourrons, je crois, bloquer ces trois rapports, étant donné la communauté d'idées dont ils procèdent.

Ces trois moyens sont : le cinéma ultra-rapide
- non plus 20 images, mais 8000 images à la seconde, la strioscopie, l'étude des sillages par voie optique grâce à une variation provoquée de l'indice de réfraction du milieu), enfin la méthode stroboscopique, qui immobilise les images et qui ne nécessite pas un phénomène rigoureusement périodique grâce à un déphasage variable dans la cadence des éclairs lumineux.

Voici la part du physicien.

Enfin, dans un quatrième rapport, le mathématicien va réapparaitre. M. FAure, chef du Département «Mécanique des Fluides» au Centre de Recherches et d'Essais de Chatou, après avoir rappelé les différents procédés de visualisation (procédés optiques, procédés par éléments traçants, procédés par traitement des surfaces), fait la critique des limites de validité de ces procédés.

Cette critique repose sur l'idée suivante, (familière aux physiciens): dès qu'on introduit un appareil ou un corps étranger, on perturbe forcément l'écoulement; même à l'échelle macroscopique, qui est celle de la mécanique des fluides, un principe d'incertitude subsiste. «Ou bien, comme le dira spirituellement M. Faune, on précise la position du point, mais alors on modifie les vitesses; ou bien on respecte l'écoulement, mais alors on ne sait plus exactement à quel point il faut rapporter la mesure. »

M. Fauns passe ainsi en revue les diverses limitations qu'apporte la mécanique des fluides à cette visualisation : masse ajoutée et poussée d'Archimède, discontinuité des pressions, distinction entre trajectoires, lignes de courant, lignes d'émission, qui ne coïncident que dans l'écoulement permanent, turbulence enfin.

C'est un mutuel et constant appui que se prêtent ainsi mathématique et physique, la perfection de la connaissance scientifique n'étant au fond que la parfaite conscience de sa limitation. 